\title{
On the Conformal Unity between Quantum Particles and General Relativity
}

\author{
Risto Raitio \\ 02230 Espoo, Finland \\ Email: risto.raitio@gmail.com
}

How to cite this paper: Raitio, R. (2017) On the Conformal Unity between Quantum Particles and General Relativity. Open Access Library Journal, 4: e3342. http://dx.doi.org/10.4236/oalib.1103342

Received: December 29, 2016

Accepted: January 16, 2017

Published: January 19, 2017

Copyright (๑) 2017 by author and Open Access Library Inc.

This work is licensed under the Creative Commons Attribution International License (CC BY 4.0).

http://creativecommons.org/licenses/by/4.0/

\begin{abstract}
I consider the standard model, together with a preon version of it, to search for unifying principles between quantum particles and general relativity. Argument is given for unified field theory being based on gravitational and electromagnetic interactions alone. Conformal symmetry is introduced in the action of gravity with the Weyl tensor. Electromagnetism is geometrized to conform with gravity. Conformal symmetry is seen to improve quantization in loop quantum gravity. The Einstein-Cartan theory with torsion is analyzed suggesting structure in spacetime below the Cartan scale. A toy model for black hole constituents is proposed. Higgs metastability hints at cyclic conformal cosmology.
\end{abstract}

\section{Subject Areas}

Particle Physics

\section{Keywords}

Preons, Standard Model, Conformal Symmetry, Torsion, Loop Quantum Gravity, Cosmology, Dark Energy, Dark Matter

\section{Introduction}

The purpose of this article is to search for unifying principles for quantum models of matter and spacetime on all possible length scales: from the tiniest distances of high energy accelerators and colliders up to galaxies and towards the radius of the universe. Even the Planck scale Gedanken experiments are considered. Cosmological developments are included with a lesser emphasisthough not less important. This task is motivated by a large number of theoretical results on the various sectors of the subject, not necessarily on the most fashionable areas of main stream research, but they are unfortunately scattered around widely in the literature. The author feels therefore that trying to collect 
some of the pieces of the puzzle together is well justified. Evidence for mathematical unity of matter and spacetime structure is indeed found, even though this work is bound to require much more effort in the future. Some of material of this note is of this author but mostly what follows is a mini review based on a personal, and partly random, selection of papers.

In the last fifty years, or more, symmetry has been the leading principle in classifying particles and their interactions. Specifically, the gauge symmetry is the basis of particle phenomenology and theory. All known particles belong to a presentation of some group, be it e.g. the Lorentz, Poincaré or an SU(N) group. The standard model (SM) of particles with its some twenty parameters describes all measured accelerator data available today. There are known limitations and problems with the standard model. Bigger problems occur when one considers astrophysical and cosmological measurements, like dark energy and dark matter. Gravity has its own known problems with quantum theory in general. String theory was a promising candidate for unifying the standard model with gravity. Unfortunately, not much progress has taken place in about fortyfive years, apart from experimentally discovering the top quark, the Higgs boson and the accelerating expansion of the universe. Have we considered in depth all possible symmetries now? Perhaps not.

One specific symmetry has long been known in special circumstances: scale, or conformal, invariance in deep inelastic scattering, magnetism and the primordial cosmic microwave background (CMB) fluctuations. ${ }^{1}$ Here I use the term conformal symmetry. The standard model is conformal symmetric if one leaves out the Higgs sector. The standard model is stable towards Planck scale but the Higgs sector is metastable. This metastability is a problem to inflationary cosmology but it fits well to conformal cyclic cosmology where the decay of the current vacuum is a prediction ending the current cycle and beginning the next one [1] [2]. The SM, including the Higgs mechanism, has been formulated as a conformal theory together with gravity [3]. Conformal symmetry changes cosmology allowing to solve classical Friedmann-Robertson-Walker (FRW) equations through big crunch-big bang transitions. Consequently, a satisfactory standard model has emerged for both small scale and large scale phenomena covering distances between $10^{-17} \mathrm{~cm}$ and $10^{28} \mathrm{~cm}$. Even dark matter and dark energy seem to find their place in this scenario.

Together with several other people, I have gone quite some time ago one step further down to preon level, for a review of early work see e.g. [4]. The term preon means here any new kind of hypothetical point particle or geometrical structure in spacetime near the Planck length scale. There is no direct experimental evidence for preons, they are rather objects of Gedanken experiments. Recently, I have reanalyzed a phenomenological preon model for quarks and leptons [5] trying to find an theoretical reference frame for it [6] [7] [8]. Unification was discussed on two levels: unification of gravitational and electromagnetic interactions only, instead of the traditional grand unification (GUT), and

${ }^{1}$ To airfoil designers the Joukowsky transformation has been the first example. 
secondly, unification of both these interactions and spacetime, i.e. unification of everything.

Unification of physics based on particle internal symmetry has been successful until recently when geometry has taken a significant role in the form of the local conformal symmetry. Gravity itself is not limited to Einstein gravity (EG). Several extra terms in the gravitational action have been studied with more and less success. In fact, a good old starting point is the gravity based on the Weyl tensor [9], which provides a unique form of the gravitational action as the square of the Weyl tensor. An almost equally old idea is, due to Einstein and Rosen, that particles themselves would be structures in pure vacuum geometry [10]. Intriguingly, Cartan introduced spinors long before spin was discovered for particles [11].

Quantization of geometry has been pursued for a few decades within a theory called loop quantum gravity (LQG) [12]. There again introducing conformal symmetry certain details of quantization have become clearer [3]. Most considerations in this note apply to the SM as well to the preon model. In the latter I wish to keep the Higgs scalar sector clean as long as possible to endorse the massless particles for conformal cosmology. On the other hand, fermion mass is considered in a generalized gravity theory, the Einstein-Cartan theory, which includes torsion [13] and brings interesting new light to fermion behavior in curved spacetime at high energy density. I give arguments for the existence of structure of spacetime at Cartan length scale, where quantum gravity is expected to begin to appear. A toy model for black hole constituents is proposed in Section 8.

This note is organized as follows. In Section 2 I briefly recall the preon model, which is discussed partly for historical reasons. In Subsection 3.1 conformal gravity and in Subsection 3.2 loop quantum gravity are summarized. In Section 4 electromagnetism is geometrized. The conformal standard model is discussed in Section 5. Section 6 is on the outer edge of this study. The Dirac field in the presence of torsion is introduced in Subsection 6.1. The massive Dirac field is discussed in Subsection 6.2. Cosmology is reviewed in Section 7. Conformal symmetry and black holes are treated in Section 8. Finally, conclusions are given in Section 9.

The presentation of the material is concise and goal oriented rather than comprehensive but an attempt is made for it to be reasonably self contained. The author feels he had to go through all the material presented in this note but the reader may find it better to start with the first and last section on first reading and save Sections 2 and 6 for later.

\section{The Preon Model}

\subsection{Conformal Preons}

The virtue of conformal symmetry is that the action for conformal gravity is defined uniquely by the Weyl tensor, described in Subsection 3.1. All particles in conformal theory are massless. Other properties of conformal theory include 
renormalizability, unitarity, and the theory is ghost-free. It has been shown to explain dark matter and energy [14] [15] [16] [17] [18]. Therefore in this note I assume zero mass preons on action level.

Requiring charge quantization $\{0,1 / 3,2 / 3,1\}$ and preon permutation antisymmetry for identical preons, one can define three preon bound states which form the first generation quarks and leptons [6] [7]

$$
\begin{aligned}
& u_{k}=\epsilon_{i j k} m_{i}^{+} m_{j}^{+} m^{0} \\
& \bar{d}_{k}=\epsilon_{i j k} m^{+} m_{i}^{0} m_{j}^{0} \\
& e=\epsilon_{i j k} m_{i}^{-} m_{j}^{-} m_{k}^{-} \\
& \bar{v}=\epsilon_{i j k} \bar{m}_{i}^{0} \bar{m}_{j}^{0} \bar{m}_{k}^{0}
\end{aligned}
$$

A binding interaction between preons is needed to make the quark and lepton bound states possible. I have at the moment no detailed form for this interaction. Its details are not expected to be of primary importance at this preliminary stage. I suppose this attractive, non-confining interaction is strong enough to keep together the charged preons but weak enough to liberate the preons at high temperature. Some more thoughts are indicated in Subsection 2.2.

A useful feature in (2.1) with two identical preons ${ }^{2}$ is that the construction provides a three-valued subindex for quark SU(3) color, as it was originally discovered [19]. In addition, the weak SU(2) left handed doublets can be read from the first two and last two lines in (2.1). The SM gauge structure can be deduced in this sense from the present preon model. One could also have preon charges $1 / 3$ and $2 / 3$ but then the index $k$ distinction between quarks and leptons in (2.1) would be lost.

One may now propose that, as far as there is an ultimate unified theory, it is a preon theory with only gravitational and electromagnetic interactions operating between preons. The strong and weak forces are generated in the early universe later when massless preons combine into quarks and leptons at lower temperature and they operate only with short range interaction within nuclei making atoms, molecules and chemistry possible. In a contracting phase of the universe processes take place in the opposite order.

The unification picture is supposed to hold in the present scheme up to the energy of about $10^{16} \mathrm{GeV}$. The electroweak interaction has the spontaneously broken symmetry phase below an energy of the order of $100 \mathrm{GeV}$ and symmetric phase above it. The electromagnetic and weak forces take separate ways at higher energies ( $100 \mathrm{GeV} \ll E \ll 10^{16} \mathrm{GeV}$ ), the latter melts away due to ionization of quarks and leptons into preons, but the former stays strong towards Planck scale, $M_{\mathrm{Pl}} \sim 1.22 \times 10^{19} \mathrm{GeV}$. Likewise the quark color interaction suffers the same destiny as the weak force. One is left with the electromagnetic and gravitational forces only at Planck scale.

The proton, neutron, electron and $v$ can be constructed of 12 preons and 12 anti-preons. The construction (2.1) is matter-antimatter symmetric on preon

${ }^{2} \mathrm{An}$ assumption appears here that the same charge preons inside quarks have the same spin $\mathrm{z}$-components. 
level, which is desirable for early cosmology. The model makes it possible to create from vacuum a universe with only matter: combine e.g. six $\mathrm{m}^{+}$, six $\mathrm{m}^{0}$ and their antiparticles to make the basic $\beta$-decay particles. Corresponding antiparticles may occur equally well.

The baryon number (B) is not conserved [20] [21] in this model: a proton may decay at Planck scale temperature by a preon rearrangement process into a positron and a pion. This is expected to be independent of the details of the preon interaction. Baryon number minus lepton number (B-L) is conserved.

Unification of gravity and electromagnetism is discussed in Section 4.

\subsection{Geometrical Preons}

The preon model described in [6] is based on a statistical black hole model in loop quantum gravity (LQG). In LQG the geometry is quantized such that the lowest area eigenvalue is zero, which allows zero mass particles in the model construction as the Brown-York energy is proportional to area. The idea that a particle can be defined in pure gravity theory was first put forward in [10]. There it was also found that a charged black hole with Reissner-Nordström metric can have zero mass.

The preons have to be kept inside the quarks and leptons using some elegant, preferably non-confining mechanism. I also want to exclude scalar, vector and spinorial self-interactions. Same sign charges should be kept inside the bound states. Therefore a possibility could be that the zero mass black hole preons would form together one single non-spherical horizon around the quark or lepton.

Unification of black hole particles (i.e. preons) and spacetime is discussed in Subsection 3.2.

\section{Gravity}

\subsection{Conformal Gravity}

Weyl introduced, while working on the geometrization of electromagnetism, a new tensor called the Weyl or conformal tensor [9] (for an introduction to conformal theories, see e.g. [22])

$$
C_{\lambda \mu \nu \kappa}=R_{\lambda \mu \nu \kappa}-\frac{1}{2}\left(g_{\lambda v} R_{\mu \kappa}-g_{\lambda \kappa} R_{\mu v}-g_{\mu v} R_{\lambda \kappa}+g_{\mu \kappa} R_{\lambda v}\right)+\frac{1}{6} R_{\alpha}^{\alpha}\left(g_{\lambda v} g_{\mu \kappa}-g_{\lambda \kappa} g_{\mu v}\right)
$$

where $R_{\lambda \mu v \kappa}$ is the Riemann tensor. Under local conformal transformation $\omega(x)$ of the metric tensor $g_{\mu v}$ of the form

$$
g_{\mu v} \rightarrow \mathrm{e}^{-2 \omega(x)} g_{\mu v}
$$

the Weyl tensor transforms simply as

$$
C_{\mu \nu \kappa}^{\lambda}(x) \rightarrow C_{\mu \nu \kappa}^{\lambda}(x)
$$

The pure local conformal gravity is based on the following action

$$
I_{W}=-\alpha_{g} \int \mathrm{d}^{4} x \sqrt{-g} C_{\lambda \mu \nu \kappa} C^{\lambda \mu v \kappa}
$$


where $\alpha_{g}$ is a dimensionless gravitational coupling constant. The action (3.4) can be written in the following simpler form [23]

$$
I_{W}=-2 \alpha_{g} \int \mathrm{d}^{4} x \sqrt{-g}\left[R_{\mu \kappa} R^{\mu \kappa}-\frac{1}{3}(R R)\right]
$$

Conformal gravity is power-counting renormalizable and unitary [16]. In $I_{W}$ there is no cosmological constant term since $\int \mathrm{d}^{4} x \sqrt{-g} g_{\mu \nu} \Lambda$ is not conformal invariant. The cosmological constant will appear later when conformal symmetry is dynamically broken and gives the dimensionful $\bar{\psi} \psi$ a non-zero vacuum expectation value.

The reason for local conformal invariance is that massless particles move on the light cone which is invariant under the 15 parameter conformal group $\mathrm{SO}(4,2)[16]$. The covering group of $\mathrm{SO}(4,2)$ is $\mathrm{SU}(2,2)$. This is generated by the 15 Dirac matrices $\left(\gamma_{5}, \gamma_{\mu}, \gamma_{\mu} \gamma_{5},\left[\gamma_{\mu}, \gamma_{v}\right]\right)$ and its fundamental representation is a fermionic field. Therefore it is natural to take fermions as the basic objects in physics. Gravity is generated by gauging the conformal symmetry on the light cone. Mass scales are to be generated dynamically by fermion bilinear condensates in the vacuum.

Functional variation of $I_{W}$ with respect to metric defines a gravitational rank two tensor $W^{\mu \nu}$ in terms of the Riemann tensor that is covariantly conserved $\nabla_{\mu} W^{\mu v}=0$ and traceless $g_{\mu \nu} W^{\mu \nu}=0$. Assuming a conformally invariant matter action $I_{M}$ (an example is discussed in the next Section 4 variation with respect to $I_{W}+I_{M}$ gives a fourth order derivative equation of motion of the form

$$
4 \alpha_{g} W^{\mu v}=T_{M}^{\mu v}
$$

where

$$
\begin{aligned}
W^{\mu v}= & 1 / 2 g^{\mu v} \partial^{\beta} \partial_{\beta}\left(R_{\alpha}^{\alpha}\right)+\partial^{\beta} \partial_{\beta} R^{\mu v}-\partial^{v} \partial_{\beta} R^{\mu \beta}-\partial^{\mu} \partial_{\beta} R^{\nu \beta} \\
& -2 R^{\mu \beta} R_{\beta}^{v}+1 / 2 g^{\mu v} R_{\alpha \beta} R^{\alpha \beta}-2 / 3 g^{\mu v} \partial^{\beta} \partial_{\beta} R_{\alpha}^{\alpha} \\
& +2 / 3 \partial^{\mu} \partial^{v} R_{\alpha}^{\alpha}+2 / 3 R_{\alpha}^{\alpha} R^{\mu v}-1 / 6 g^{\mu v}\left(R_{\alpha}^{\alpha}\right)^{2}
\end{aligned}
$$

By defining the left hand side of (3.6) as the energy-momentum tensor $T_{\text {gravity }}^{\mu v}$ we can write (3.6) in the form

$$
T_{\text {gravity }}^{\mu v}+T_{M}^{\mu v}=0
$$

We see that gravity and matter sectors are on equal footing and the total energy-momentum tensor of the universe is zero.

The connection between Einstein gravity and conformal gravity is that the solutions of the former are solutions of the latter [24] [25]. However, conformal gravity has more general solutions to be explored. From these the Einstein solutions can be chosen by imposing a certain Neumann boundary condition on the metric at the future boundary. The boundary condition eliminates ghosts and the theory becomes an Einstein theory with a cosmological constant.

As a final piece of support to conformal invariance it can be mentioned that the high energy limit of all non-trivial renormalizable field theories is comformally invariant [26]. 


\subsection{Loop Quantum Gravity}

A statistical physics model for quantum black holes has been presented in [7]. It is based on Brown-York energy $E$ for an area $A$ of a horizon: $E=a c^{2} A / 8 \pi G$ where $a$ is the constant proper acceleration of an observer on the stretched horizon. In LQG the area eigenvaleus are

$$
A=\gamma l_{\mathrm{Pl}}^{2} \sum_{p} \sqrt{j_{p}\left(j_{p}+1\right)}
$$

where the sum is over punctures $p$ of the spin network, $l_{\mathrm{pl}}$ is the Planck length, $\gamma$ is the Barbero--Immirzi parameter and the values of $j_{p}$ are half integral. The spin number $j_{p}$ describes the size of the quanta of space [27]. For comprehensive treatments of quantum geometry and black holes see e.g. [28].

Among the problems in LQG is finding quantization without quantum anomalies. A second difficulty is making contact with the semiclassical physical picture of gravity. The existence of Planck scale sets restrictions in going to the continuum limit. If one adds points to the spin network to refine it, the continuum approximation of volumes and areas does not get better, one just adds volume to the spacetime as the area eigenvalue has a minimum value. In a conformal theory there is no length scale available and it is possible to improve the situation. Thirdly, though the theory is discrete and therefore finite, a finite renormalization is needed to separate the lower energy physics from the Planck scale features [3].

Conformal invariance helps in all the above difficulties. In a conformal theory spin networks can be defined which can be indefinitely refined to arbitrary precision. In the renormalization problem no counter terms are needed in spacing dependent renormalization.

The geometric operators of area of a surface and the volume of a region can be generalized to their conformal invariant counterparts which are the same as before but now without factors of Planck length to the relevant power. Thus a conformal geometry of a spin network can be defined [3].

\section{Geometrization of Electromagnetism}

Conformal invariance is also important for understanding the geometrization of other interactions. Metrication of electromagnetism coupled to a Dirac field $\psi(x)$ is given in [29] (see also [30]).

The generalized Dirac action is

$$
I_{D}=\int \mathrm{d}^{4} x \sqrt{-g} i \bar{\psi} \gamma^{c} e_{c}^{\mu}(x)\left(\partial_{\mu}+\tilde{\Gamma}_{\mu}\right) \psi+\text { h.c. }
$$

where the $\gamma^{a}$ are the Dirac matrices, $e_{c}^{\mu}(x)$ is a vierbein defined by $g_{\mu v}=e_{\mu}^{a} e_{v}^{b} \eta_{a b}$ (solving this for $\eta_{\mu v}$, the vierbein vectors are seen to diagonalize the metric tensor) and

$$
\tilde{\Gamma}_{\mu}(x)=\frac{1}{8}\left[\gamma_{a}, \gamma_{b}\right]\left(e_{\nu}^{b} \partial_{\mu} \mathrm{e}^{a v}+e_{\lambda}^{b} \tilde{\Gamma}_{\nu \mu}^{\lambda} \mathrm{e}^{a v}\right)
$$

which is obtained from the generalized connection

$$
\tilde{\Gamma}_{\mu v}^{\lambda}=\Lambda_{\mu v}^{\lambda}+W_{\mu v}^{\lambda}
$$


where $\Lambda_{\mu \nu}^{\lambda}$ and $W_{\mu \nu}^{\lambda}$ are the Levi-Civita and Weyl connections, respectively

$$
\begin{aligned}
& \Lambda_{\mu \nu}^{\lambda}=\frac{1}{2} g^{\lambda \alpha}\left(\partial_{\mu} g_{v \alpha}+\partial_{\nu} g_{\mu \alpha}-\partial_{\alpha} g_{\mu v}\right) \\
& W_{\mu \nu}^{\lambda}=-g^{\lambda \alpha}\left(g_{v \alpha} A_{\mu}+g_{\mu \alpha} A_{\nu}-g_{v \mu} A_{\alpha}\right)
\end{aligned}
$$

where $A_{\alpha}$ is the electromagnetic potential. It turns out that the covariant derivative of the metric in (4.1) is non-zero, $\tilde{\nabla}_{\lambda} g^{\mu v}=-2 g^{\mu v} A_{\lambda}$, and therefore parallel transport is path dependent and the theory is untenable.

Secondly, the Weyl connection drops out from the generalized Dirac action and therefore does not provide geometrization of electromagnetism. Replacing $W_{\mu \nu}^{\lambda}$ by

$$
\mathcal{W}_{\mu \nu}^{\lambda}=-\frac{2 i}{3} g^{\lambda \alpha}\left(g_{v \alpha} A_{\mu}+g_{\mu \alpha} A_{\nu}-g_{v \mu} A_{\alpha}\right)
$$

and $\partial_{\mu}$ by $\partial_{\mu}-2 A_{\mu}$ in (4.1) one ends up with a satisfactory action

$$
I_{D}=\int \mathrm{d}^{4} x \sqrt{-g} i \bar{\psi} \gamma^{c} e_{c}^{\mu}(x)\left(\partial_{\mu}+\Gamma_{\mu}-i A_{\mu}\right) \psi
$$

where $\gamma^{\mu}(x)=e_{a}^{\mu}(x) \gamma^{a}$ and

$$
\Gamma_{\mu}(x)=1 / 8\left(\left[\gamma^{v}(x), \partial_{\mu} \gamma_{\nu}(x)\right]-\left[\gamma^{v}(x), \gamma_{\sigma}(x)\right] \Gamma_{\mu \nu}^{\sigma}\right)
$$

The action $I_{D}$ is locally invariant under both gauge and conformal transformations $\omega(x)$

$$
\begin{aligned}
& \psi(x) \rightarrow \mathrm{e}^{-3 \omega(x) / 2} \psi(x) \\
& g_{\mu v} \rightarrow \mathrm{e}^{-2 \omega(x)} g_{\mu v} \\
& e_{\mu}^{\alpha} \rightarrow \mathrm{e}^{\omega(x)} e_{\mu}^{\alpha} \\
& A_{\mu}(x) \rightarrow A_{\mu}(x)
\end{aligned}
$$

One sees that $A_{\mu}(x)$ does not transform at all. The equation of motion for preons, without preon-preon interactions, is (3.8).

\section{Conformal Standard Model}

It is possible to couple massless particles to conformal gravity. One can also couple the massless standard model, or even the complete standard model in a way in which the Higgs boson acquires mass in the gauge fixed conformal theory [3] [31]. When the gauge is fixed the Planck scale is determined and so further the Higgs mass and the masses of all other SM particles, determined by the dimensionless constants of the theory.

The SM total Lagrangian can be written as a sum of the gravitational and matter terms

$$
\mathcal{L}_{T}=\mathcal{L}_{G R}\left(g^{c}\right)+\mathcal{L}_{M}\left(g^{c}, \psi^{M} / \phi^{d}\right)
$$

where $g_{a b}^{c}=\phi^{2} g_{a b}, \psi^{M}$ are the matter fields, $\phi$ is the Brans-Dicke scalar field [32] and $d$ is a suitable power, like 1 or $3 / 2$, to ensure the conformal invariance of matter fields. The equations of motion imply that the stress tensor of the matter fields is traceless. All the SM particles can now be incorporated 
without mass. The Higgs field is introduced as a doublet $h^{\alpha}, \alpha=1,2$. The action is now in terms of conformal variables

$$
S=\int \mathrm{d}^{4} x \sqrt{-g_{c}}\left[-g^{a b(c)} D_{\alpha} h^{c \dagger} D_{b} h^{c}-\frac{\lambda}{4}\left(h^{c \dagger} h^{c}-\alpha^{2}\right)^{2}+\frac{1}{4} \lambda^{\prime}+\mathcal{L}_{S M}\left(g^{c}, \psi^{c}, A_{a}\right)\right]
$$

where the $\lambda$ and $\lambda^{\prime}$ are coupling constants and $A_{a}$ refers to the gauge fields of the $\mathrm{SM}$, in the simplest case the $\mathrm{SU}(2) \times \mathrm{U}(1)$ fields.

Choosing the gauge $\phi(x)=\phi_{0}$ one gets the dimensionful parameters in terms of $\phi_{0}$

$$
G=1 / 8 \pi \phi_{0}^{2}, \Lambda / 4 \pi G=\lambda^{\prime} \phi_{0}^{4}
$$

where $\Lambda$ is the cosmological constant (as usual, its value is nowhere near the measured value). The Higgs mass and vacuum expectation value are

$$
m_{\text {Higgs }}^{2}=\lambda \alpha^{2} \phi^{2},\left\langle h^{\dagger} h\right\rangle=\alpha^{2} \phi^{2}
$$

This is a brief summary of a conformal invariant theory of gravity coupled to the SM that can be quantized by the LQG techniques [3].

\section{Torsion}

\subsection{Dirac Field in the Presence of Torsion}

In this section I want to emphasize another kind of treatment of conformal gravity and the Dirac field, which can be a quark, lepton, or preon. In GR, one has to discuss torsion arising from rotations and translations of the Poincaré group, just as energy gives rise to curvature [33] [34]. Experimentally there is no evidence for torsion. It will be shown below that constraints coming from the antisymmetry of the spin do not yield complete antisymmetry of torsion but cause constraints to the metric. In particular, the spinorial self-interactions are absent. If, in addition, no scalar interactions are allowed for spinors their zero masses are well protected. In the case of preons, this sector of the model joins smoothly to the conformal SM when the energy or temperature becomes so low as to allow quark and lepton bound states.

The Riemann-Cartan geometry with metric and torsion is defined in terms of the metric tensor $g_{\mu v}$ and a metric-compatible connection $\Gamma_{\mu \nu}^{\lambda}$, which are independent. A connection is metric compatible if the covariant derivative of the metric with respect to that connection is everywhere zero. Given any connection the torsion tensor is defined as

$$
Q_{\mu v}^{\lambda}=\Gamma_{\mu \nu}^{\lambda}-\Gamma_{v \mu}^{\lambda}=\Gamma_{[\mu v]}^{\lambda}
$$

The torsion tensor is antisymmetric in its lower indices. A symmetric connection is known as torsion-free.

The most general conformal transformation for the metric and torsion are with $\phi=\log \sigma$

$$
\begin{aligned}
& Q_{\rho \alpha}^{\sigma} \rightarrow Q_{\rho \alpha}^{\sigma}+q\left(\delta_{\rho}^{\sigma} \partial_{\alpha} \phi-\delta_{\alpha}^{\sigma} \partial_{\rho} \phi\right) \\
& g_{\alpha \beta} \rightarrow \sigma^{2} g_{\alpha \beta}
\end{aligned}
$$


where $q$ is the conformal charge. It is seen in (6.2) that the conformal transformation of the torsion is a transformation of its trace vector $Q_{\alpha} \rightarrow Q_{\alpha}+3 q \partial_{\alpha} \phi$. The vierbein transforms as follows

$$
e_{\alpha}^{k} \rightarrow \sigma e_{\alpha}^{k}
$$

The Dirac field conformal transformation is

$$
\psi \rightarrow \sigma^{-3 / 2} \psi, \bar{\psi} \rightarrow \sigma^{-3 / 2} \bar{\psi}
$$

Let us introduce the modified metric-torsional curvature tensor with the Riemann curvature tensor $R_{\alpha \beta \mu v}$

$$
M_{\alpha \beta \mu v}=R_{\alpha \beta \mu v}+\frac{1-q}{3 q}\left(Q_{\beta} Q_{\alpha \mu v}-Q_{\alpha} Q_{\beta \mu v}\right)
$$

whose irreducible part is

$$
T_{\alpha \beta \mu v}=M_{\alpha \beta \mu v}-\frac{1}{2}\left(M_{\alpha[\mu} g_{v] \beta}-M_{\beta[\mu} g_{v] \alpha}\right)+\frac{1}{12} M\left(g_{\alpha[\mu} g_{v] \beta}-g_{\beta[\mu} g_{v] \alpha}\right)
$$

and it is conformally covariant. The commutator of covariant derivatives obeys the equation

$$
\left[D_{\mu}, D_{v}\right] \psi=Q_{\mu v}^{\alpha} D_{\alpha} \psi+G_{\mu v} \psi
$$

The conformal transformation for torsion is not uniquely defined [35]. The most general invariant obtainable from $T_{\alpha \beta \mu v}$ in (6.6) is the expression

$$
a T^{\alpha \beta \mu v} T_{\alpha \beta \mu v}+b T^{\alpha \beta \mu v} T_{\mu v \alpha \beta}+c T^{\alpha \beta \mu v} T_{\alpha \mu \beta v}
$$

with the parameters $a, b$ and $c$. Define the quantity $P_{\alpha \beta \mu v}$ as follows

$$
P_{\alpha \beta \mu v}=a T_{\alpha \beta \mu v}+b T_{\mu v \alpha \beta}+\frac{c}{4}\left(T_{\alpha \mu \beta v}-T_{\beta \mu \alpha v}+T_{\beta v \alpha \mu}+T_{\alpha \nu \beta \mu}\right)
$$

(6.9) is antisymmetric in the first and second pair of indices, irreducible and conformally covariant. This reduces to the form $T^{\alpha \beta \mu v} P_{\alpha \beta \mu v}$ and the most general Dirac action is

$$
S=\int \mathrm{d}^{4} x \sqrt{-g}\left[k T^{\alpha \beta \mu v} P_{\alpha \beta \mu v}+L_{\text {matter }}\right]
$$

with $k$ the gravitational constant. By variation one gets

$$
\begin{gathered}
4 k\left[D_{\rho} P^{\alpha \beta \mu \rho}+Q_{\rho} P^{\alpha \beta \mu \rho}-\frac{1}{2} Q_{\rho \theta}^{\mu} P^{\alpha \beta \rho \theta}\right. \\
\left.-\left(\frac{1-q}{3 q}\right)\left(Q_{\rho} P^{\rho[\alpha \beta] \mu}-\frac{1}{2} Q_{\sigma \rho \theta} g^{\mu[\alpha} P^{\beta] \sigma \rho \theta}\right)\right]=S^{\mu \alpha \beta} \\
2 k\left[P^{\theta \sigma \rho \alpha} T_{\theta \sigma \rho}^{\mu}-\frac{1}{4} g^{\alpha \mu} P^{\theta \sigma \rho \beta} T_{\theta \sigma \rho \beta}+P^{\mu \sigma \alpha \rho} M_{\sigma \rho}\right. \\
+\left(\frac{1-q}{3 q}\right)\left(D_{v}\left(2 P^{\mu \rho \alpha v} Q_{\rho}-g^{\mu \alpha} P^{\nu \theta \rho \sigma} Q_{\theta \rho \sigma}+g^{\mu v} P^{\alpha \theta \rho \sigma} Q_{\theta \rho \sigma}\right)\right. \\
\left.\left.+Q_{v}\left(2 P^{\mu \rho \alpha v} Q_{\rho}-g^{\mu \alpha} P^{v \theta \rho \sigma} Q_{\theta \rho \sigma}-P^{\mu v \rho \sigma} Q_{\rho \sigma}^{\alpha}\right)\right)\right]=\frac{1}{2} T^{\alpha \mu}
\end{gathered}
$$

where $S^{\rho \mu \nu}$ and $T^{\mu \nu}$ are the spin and energy density tensors of the matter conformal field. Here both Weyl equations and this new set of equations 
describe how energy and spin are the source of an intertwined combination of both curvature and torsion. This fact will be interesting for Dirac matter.

The Dirac action is

$$
S=\int \mathrm{d}^{4} x\left[L_{\text {gravity }}+\frac{i}{2}\left(\bar{\psi} \gamma^{\rho} D_{\rho} \psi-D_{\rho} \bar{\psi} \gamma^{\rho} \psi\right)\right]|e|
$$

where $e=\operatorname{det} e_{i}^{a}$. By variation of the action one gets the antisymmetric spin and traceless energy densities

$$
\begin{gathered}
S_{\mu \alpha \beta}=\frac{1}{4} \varepsilon_{\mu \alpha \beta \rho} \bar{\psi} \gamma^{\rho} \gamma \psi \\
T_{\mu \alpha}=\frac{i}{2}\left(\bar{\psi} \gamma_{\mu} D_{\alpha} \psi-D_{\alpha} \bar{\psi} \gamma_{\mu} \psi\right)
\end{gathered}
$$

and the massless matter field equations are

$$
i \gamma^{\mu} D_{\mu} \psi+\frac{i}{2} Q_{\mu} \gamma^{\mu} \psi=0
$$

To see the effects of the complete antisymmetry of the spin on the structure of the field equations one should rewrite the field equations as follows

$$
\begin{aligned}
& 4 k\left[D_{\rho} P^{\alpha \beta \mu \rho}+Q_{\rho} P^{\alpha \beta \mu \rho}-\frac{1}{2} Q_{\rho \theta}^{\mu} P^{\alpha \beta \rho \theta}-\left(\frac{1-q}{3 q}\right)\left(Q_{\rho} P^{\rho[\alpha \beta] \mu}\right)\right] \\
& =\frac{1}{4} \varepsilon^{\mu \alpha \beta \rho} \bar{\psi} \gamma_{\rho} \gamma \psi \\
& \quad 2 k\left[P^{\theta \sigma \rho \alpha} T_{\theta \sigma \rho}^{\mu}-\frac{1}{4} g^{\alpha \mu} P^{\theta \sigma \rho \beta} T_{\theta \sigma \rho \beta}+P^{\mu \sigma \alpha \rho} M_{\sigma \rho}\right. \\
& \left.\quad+\left(\frac{1-q}{3 q}\right)\left(D_{v}\left(2 P^{\mu \rho \alpha \nu} Q_{\rho}\right)+Q_{\nu}\left(2 P^{\mu \rho \alpha \nu} Q_{\rho}-P^{\mu \nu \rho \sigma} Q_{\rho \sigma}^{\alpha}\right)\right)\right] \\
& =\frac{i}{4}\left(\bar{\psi} \gamma^{\alpha} D^{\mu} \psi-D^{\mu} \bar{\psi} \gamma^{\alpha} \psi\right)
\end{aligned}
$$

with the massless matter field equations (6.16).

In Weyl gravity there is no more a completely antisymmetric torsion and there are additional constrictions on the curvature tensor. This happens because both field equations for the spin and energy couple to both torsion and curvature so that the complete antisymmetry of the spin is partly imposed on torsion and partly on the curvature. We may decompose torsional terms away from the torsionless ones in all curvatures and derivatives. Thereafter all curvatures and derivatives are written in terms of purely metric curvature and derivatives given by the Weyl conformal tensor $C_{\alpha \beta \mu \nu}$ and the Levi-Civita derivative $\nabla_{\mu}$ plus contributions due to torsion $Q_{\mu \alpha \sigma}$ decomposable in its three components according to

$$
Q_{\mu \alpha \sigma} \equiv T_{\mu \alpha \sigma}+\epsilon_{\mu \alpha \sigma \rho} W^{\rho}+\frac{1}{3}\left(g_{\mu \alpha} Q_{\sigma}-g_{\mu \sigma} Q_{\alpha}\right)
$$

where $T_{\mu \alpha \sigma}$ is the non-completely antisymmetric irreducible part and $W^{\alpha}$ is the axial vector dual of the completely antisymmetric irreducible part of the torsion. The Dirac equation, in Einstein-type of gravity, is of the general form 


$$
i \gamma^{\mu} \nabla_{\mu} \psi-\frac{3}{4} W_{\mu} \gamma^{\mu} \gamma \psi=0
$$

In Weyl-type of gravity there is no possibility to substitute torsion with the spin of the spinors and there are no longer non-linear self-interactions in the spinor field equations. The Dirac equation is linear even in the presence of torsion.

The conclusion from all the above analysis of torsion in GR is that the nonlinear self-interactions of Dirac matter fields are absent.

\subsection{Massive Dirac Field in the Presence of Torsion}

A Dirac field with mass is a non-conformal theory, but it is a very interesting case and is discussed briefly. The Einstein-Cartan (EC) [11] [36], or EinsteinCartan-Kibble-Sciama (ECKS) [37] [38] [39] theory of gravity is a natural extension of GR to include matter with spin as is necessary to take into account the local gauge invariance with respect to the full Poincare group. This produces torsion. The effect of torsion occur only at very high density of matter, much larger than the density of nuclear matter. Torsion modifies Dirac-Kerr-Newman ring singularity by a non-singular toroidal structure with the outer radius of the Compton wave length size and inner radius of the Cartan size (see (6.31) below). The Cartan size may introduce an effective UV cutoff for fermionic quantum field theory.

The dynamical variables in Einstein-Cartan theory are the vierbein $e_{a}^{i}$ and the spin connection

$$
\omega_{b k}^{a}=e_{j}^{a}\left(\partial_{k} e_{b}^{j}+\Gamma_{i k}^{j} e_{b}^{i}\right)
$$

where $\Gamma_{i k}^{j}$ is the affine connection or Christoffel symbol. It is asymmetric in the lower indices and its antisymmetric part is the torsion tensor $S_{j k}^{i}=\Gamma_{[j k]}^{i}$. The notation [] means antisymmetrization. The dynamical energy-momentum density is defined by the variation of the Lagrangian density of matter $\mathcal{L}_{m}$ with respect to vierbein

$$
\Theta_{i}^{a}=\delta L_{m} / \delta \omega_{i}^{a b}
$$

The variation with respect to spin connection $\omega_{i}^{a b}$ defines the dynamical spin density

$$
\sum_{a b}^{i}=2 \delta \mathcal{L}_{m} / \delta \omega_{i}^{a b}
$$

The ECKS Lagrangian density is

$$
\mathcal{L}=\mathcal{L}_{M}-e R / 16 \pi G
$$

where $e=\operatorname{det} e_{i}^{a}, \quad R=R_{i}^{a} e_{a}^{i}$ is the Ricci scalar and $G$ the gravitational constant $(c=1)$. This is the simplest of various theories of gravity with torsion. The Cartan equation relates locally the torsion of spacetime to the spin density (6.21)

$$
e\left(S_{a b}^{i}-S_{a} e_{b}^{i}+S_{b} e_{a}\right)=-4 \pi G \sum_{a b}^{i}
$$

where $S_{i}=S_{i k}^{k}$ is the torsion vector coming from the variation of the ECKS action under spin connection. Combining Einstein equation and (6.25) yields 


$$
G_{i k}=8 \pi G T_{i k}+U_{i k}
$$

where $G_{i k}=R_{i k}-1 / 2 R g_{i k}$ is the Einstein tensor and $T_{i k}=2 / e \delta \mathcal{L}_{M} / \delta g^{i k}$ is the metric energy-momentum tensor. The tensor $U_{i k}$

$$
\begin{aligned}
U_{i k}= & -\left(S_{i j}^{l}+2 S_{(i j)}^{l}\right)\left(S_{k l}^{j}+2 S_{(k l)}^{j}\right)+4 S_{i} S_{k} \\
& +1 / 2\left(G_{i k} S^{m j l}+2 S^{(j l) m}\right)\left(S_{l j m}+2 S_{(j m) l}\right)-2 g_{i k} S^{j} S_{j}
\end{aligned}
$$

where () denotes symmetrization, is quadratic in $\sum_{i j}^{k}$. The torsion is zero in GR and (6.26) reduces to Einstein equations.

The Cartan equation (6.25) is a linear relation and torsion is proportional to spin density. Therefore the torsion is zero outside material bodies. This makes detection of torsion difficult. The torsion field of ECKS theory does not propagate, unlike curvature.

The relativistic Dirac Lagrangian density in curved spacetime is

$$
\begin{aligned}
\mathcal{L}_{\psi}= & 1 / 2 i \hbar e\left(\bar{\psi} \gamma^{i} \partial_{i} \psi-\partial_{i} \bar{\psi} \gamma^{i} \psi\right)-1 / 2 i \hbar e\left(\bar{\psi}\left(\gamma^{i} \Gamma_{i}+\Gamma_{i} \gamma^{i}\right) \psi\right) \\
& -q e \bar{\psi} \gamma^{i} \psi A_{i}-m e \bar{\psi} \psi
\end{aligned}
$$

where the $\gamma^{i}$ are the Dirac matrices, $m$ is the mass, $q$ the charge of the particle and $A$ is the electromagnetic potential $(c=1)$. The spinor connection is $\Gamma_{i}=-1 / 4 \omega_{a b i} \gamma^{a} \gamma^{b}$. The spin density corresponding to (6.28) is totally antisymmetric

$$
\sum^{i j k}=1 / 2 i \hbar e \bar{\psi} \gamma^{i} \gamma^{j} \gamma^{k} \psi
$$

This spin density (6.29) does not depend on $m$ or $q$. It remains the same if one includes the weak and strong interactions for the fermions. Substituting the spin density (6.29) into (6.28) one introduces the Heisenberg-Ivanenko fourfermion self-inteaction term in the Lagrangian density

$$
\mathcal{L}_{S}=3 / 2 \pi G e \hbar^{2}\left(\bar{\psi} \gamma^{i} \gamma^{5} \psi\right)\left(\bar{\psi} \gamma_{i} \gamma^{5} \psi\right)
$$

If one assumes the simplest possible fermion system, namely a point particle or a system of point particles, it turns out that there exist no solutions for the spinor field, i.e. $\psi=0$. The same happens for a fermionic string. Thus torsion in ECKS theory does not make it possible for a Dirac field to form point or string configurations. Torsion determines the minimal spatial extension $d$ of a spinor field. The size comes from the condition that the repulsive four-fermion self-interaction term balances the gravitationally attractive mass term in (6.28). The energy-momentum tensor in (6.28) is of the order of $m\left|\psi^{2}\right|$, the spin density $\hbar\left|\psi^{2}\right|$ and the wave function $\psi \sim d^{3 / 2}$. Therefore the size is of the order of the Cartan radius $r_{C}$ defined by

$$
m / r_{C}^{3} \sim G\left(\hbar / r_{C}^{3}\right)^{2}
$$

For an electron, $r_{C} \sim 10^{-25} \mathrm{~cm}$, which is much less than its Compton wave length $h / m \sim 10^{-10} \mathrm{~cm}$. For heavier fermions, $r_{C}$ is below $\sim 10^{-27} \mathrm{~cm}$. If the ECKS theory is correct an effective UV cutoff for quantum field theory would be of the order of $r_{C}$. If GR is correct the cutoff would be much smaller Planck 
scale $l_{\mathrm{Pl}}$.

These results imply that the Dirac wave function of an electron forms a nonsingular form of spacetime structure of a toroid which has the outer radius of the electron Compton wave length and the inner radius of its Cartan radius. This is valid both for charged and uncharged leptons. The weak interactions do not change the situation in any significant amount. The toroid structure works also for quarks for which asymptotic freedom holds at distances $\sim r_{C}$. Free fermions in the ECKS theory must therefore extend in two spatial dimensions at least on the scale of their Cartan radii. The Cartan density for an electron, $\rho_{C} \sim m_{e} / r_{C}^{3} \sim 10^{49} \mathrm{~g} / \mathrm{ccm}$ approximates the order of the maximum density of matter composed of standard model particles. Gravitational collapse of fermionic matter cannot create a singularity even if an event horizon is formed. Somewhat surprisingly, this corresponds a minimum mass of a black hole of the order of $10^{43} \mathrm{GeV}$ which is a way above the $M_{\mathrm{Pl}} \cdot{ }^{3}$

Finally, for the cosmological constant it is derived in [40], using the type of four-fermion interaction (6.30)

$$
\Lambda=\frac{3}{16 M_{\mathrm{Pl}}^{4}}\left(\bar{\psi} \gamma_{i} \gamma^{5} \psi\right)\left(\bar{\psi} \gamma^{i} \gamma^{5} \psi\right)
$$

This $\Lambda$, induced by torsion, depends on spinor fields and is not constant in time. If the spinor fields can form a condensate the vacuum expectation value of $\Lambda$ behaves like a cosmological constant. Quark fields in quantum chromodynamics form a condensate with a vacuum expectation value $\approx-(230 \mathrm{MeV})^{3}$. This energy scale is only about ten times larger than the observed $\Lambda$ value.

\section{Cyclic Conformal Cosmology}

The running standard model quartic Higgs coupling $\lambda$ switches sign from positive to negative value when the vacuum expectation value of the Higgs field $h$ exceeds $10^{10-12} \mathrm{GeV}$, assuming that no new physics below the Planck scale changes the situation [41]. The measured values of the Higgs and top quark masses are essential for this result: the electroweak vacuum is metastable being maintained by a low energy barrier of height $\left(10^{10-12} \mathrm{GeV}\right)^{4}$, well below the Planck density. This means that the universe has a finite lifetime before decaying into a contracting phase caused by a large negative potential energy density. On the theoretical side, this is a problem for the inflationary model. In short, because of fine tuned initial conditions the past of our universe is unlikely and its future precarious [1].

But for the metastable Higgs there is a better solution, cyclic cosmology [2]. According to the cyclic picture, the vacuum is required to be metastable in order for the current phase of accelerated expansion to end and for a big crunch/big bang transition to occur enabling a new cycle to begin. It is important that scalar fields exist that tunnel from the current vacuum of positive potential energy density to a phase with negative potential energy density which decreases deeper

${ }^{3}$ This result is intuitively strange. 
with increasing field magnitude. For the cyclic model, this behavior not only is part of the future but also part of our distant past leading to the most recent bounce, the big bang.

In [2] Bars, Steinhardt and Turok construct a theoretical model that supports all known accelerator and satellite physics and describes the evolution of the Higgs through the big bounce. A major question is whether there exist solutions that will return the Higgs to the metastable vacuum after each big crunch/big bang transition.

The guiding principle of the model is conformal symmetry. The Weyl invariant action $S=\int \mathrm{d}^{4} x \mathcal{L}(x)$ to describe gravity and the standard model is

$$
\begin{aligned}
\mathcal{L}(x)= & \sqrt{-g}\left[\frac{1}{12}\left(\phi^{2}-2 h^{\dagger} h\right) R(g)+g_{\mu \nu}\left(\frac{1}{2} \partial_{\mu} \phi \partial_{\nu} \phi-D_{\mu} h^{\dagger} D_{v} h\right)\right. \\
& \left.-\left(\frac{\lambda}{4}\left(h^{\dagger} h-\mu^{2} \phi^{2}\right)^{2}+\frac{\lambda^{\prime}}{4} \phi^{4}\right)+L_{S M}\right]
\end{aligned}
$$

The term $L_{S M}$ invludes the terms of the standard model Lagrangian except for the kinetic and self-interaction terms of the Higgs doublet $h(x)$ which are explicitly indicated in (7.1). The scalar field $\phi(x)$ is a singlet under $\mathrm{SU}(2) \times$ $\mathrm{U}(1)$ and it couples only to the Higgs field but not to the other fields of the SM. $\phi$ looks like a ghost but one can choose a Weyl gauge $\Omega(x)$ such that $\phi$ is constant, $\phi_{0} \equiv \phi_{c}$, and therefore is eliminated as a physical degree of freedom. This gauge is called c-gauge. $\mu$ is a parameter, $10^{-17}$ in Planck units, and it determines the Higgs vacuum expectation value and the Higgs mass. Both $\phi$ and $h$ are conformally coupled scalars and the coefficient $1 / 12$ is due to local Weyl symmetry. There is a relative minus sign between $h$ and $\phi$ kinetic energy terms and the Ricci scalar couplings in order to have $h$ as the proper physical scalar with conformal symmetry requirements.

The action (7.1) is invariant under Weyl transformations by a local function $\Omega(x)$ as follows

$$
g_{\mu \nu} \rightarrow \Omega^{-2} g_{\mu v}, s \rightarrow \Omega s, \psi_{q, l} \rightarrow \Omega^{-3 / 2} \psi_{q, l}, A_{\mu}^{\gamma, W, Z, g} \rightarrow A_{\mu}^{\gamma, W, Z, g}
$$

where $s$ is the scalar field $h$ or $\phi$.

In the gauge $\phi=\phi_{0}$ the physical parameters can be expressed in terms pf $\phi_{0}$ as follows

$$
\begin{gathered}
3 / 4 \pi G=\phi_{0}^{2}, \Lambda / 4 \pi G=\lambda^{\prime} \phi_{0}^{4} \\
h_{0}^{\dagger} h_{0}=\omega^{2} \phi_{0}^{2} \equiv v^{2} / 2
\end{gathered}
$$

The action (7.1) defines a conformally invariant homogenous and isotropic Friedman-Robertson-Walker (FRW) universe [42]

$$
\begin{aligned}
S= & \int \mathrm{d} \tau(-1 / 2 e)\left[\left[\left(\partial_{\tau}(a \phi)\right)^{2}+\left(\partial_{\tau}(a h)\right)^{2}\right]\right. \\
& \left.-e\left[a^{4} V(\phi, h)+\rho_{r}+C \sqrt{\rho_{r}} a^{2} h^{2}+\mathcal{K}\left(\phi^{2}-h^{2}\right) a^{2}\right]\right]
\end{aligned}
$$

where $\tau$ is the conformal time, $e$ is the lapse function, $C$ is a dimensionless constant, $\mathcal{K}$ is the spatial curvature and $V(t \phi, t h)=t^{4} V(\phi, h)$ describes the 
Higgs potential. The gauge bosons and fermions are treated as a radiation fluid at temperature $T$ which induces a term like $T^{2} h^{\dagger} h \sim \sqrt{\rho_{r}} a^{2} h^{2}$ for the effective Higgs field potential. The radiation density in Einstein frame (where Ricci scalar is not multiplied by the scalar field) is $\rho_{r} / a_{E}^{4} \propto T^{4}$ and $\rho_{r}$ is a constant.

Cosmological variables and gauges are briefly discussed, in $\gamma$-gauge ( $a \equiv a_{\gamma}=1$ ), and there is no cosmological singularity, the Higgs potential is purely quartic $V\left(\phi_{\gamma}, h_{\gamma}=1 / 4\left(\lambda h_{\gamma}^{4}+\lambda^{\prime} \phi_{\gamma}^{4}\right)\right)$ and the dynamics of the universe is described smoothly by the fields $\phi_{\gamma}$ and $h_{\gamma}$. The authors of [1] focus on studying cyclic solutions taking $\lambda^{\prime}$ to be negative and smaller than all other scales. An effect is needed in the cyclic model where $\lambda^{\prime}$ would be replaced by a field, the Higgs, that tunnels from a small positive energy density, the current dark energy density, to a negative value to change development from expansion to contraction. For the running coupling $\lambda(h / \phi)$ the form obtained in [41] is assumed in a simplified form

$$
\lambda(h / \phi)=\lambda_{0}\left[1-\epsilon \log (h / \omega \phi)^{2}\right]
$$

where $\lambda_{0}$ is to fit the Higgs mass in today's Higgs vacuum at $h / \phi=\omega \approx 10^{-17}$ and $\epsilon$ is chosen to make the quartic coupling pass negative at $h_{c} \approx 10^{12} \mathrm{GeV}$.

The authors are now able to conclude having found a band of continuous solutions that undergo acceptable repeated cycles of expansion and contraction as illustrated in detail in [2].

\section{Conformal Symmetry and Black Holes}

The conformal action is (3.4). The general static, spherically symmetric solutions of (3.8) is [43]

$$
\mathrm{d} s^{2}=-V(\rho) \mathrm{d} \tau^{2}+V(\rho)^{-1} \mathrm{~d} \rho^{2}+\rho^{2}\left(\mathrm{~d} \Theta^{2}+\sin ^{2} \Theta \mathrm{d} \Phi^{2}\right)
$$

where the function $V$ is

$$
V(\rho)=1-\beta(2-3 \beta \gamma) / \rho-3 \beta \gamma+\gamma \rho-k \rho^{2}
$$

where $\beta, \gamma$ and $k$ are constants. By analytically continuing (8.1) as follows

$$
\tau=i t, \rho=i r, \Theta=i \theta, \Phi=\phi, \beta=-i b, \gamma=i c
$$

which gives

$$
\mathrm{d} s^{2}=-V(r) \mathrm{d} t^{2}+V(r)^{-1} \mathrm{~d} r^{2}+r^{2}\left(\mathrm{~d} \theta^{2}+\sinh 2 \theta \mathrm{d} \phi^{2}\right)
$$

where $V(r)$ is now given by

$$
V(r)=-1-b(2-3 b c) / r+3 b c+c r-k r^{2}
$$

For certain values of the parameters (8.4) is a black hole line element. The metric on the spacelike surfaces of constant $r$ and $t$ on the event horizon is

$$
\mathrm{d} \sigma^{2}=r^{2}\left(\mathrm{~d} \theta^{2}+\sinh ^{2} \theta \mathrm{d} \phi^{2}\right)
$$

This metric describes a non-compact hyperbolic two-space $H^{2}$ with constant 
negative curvature. The $(\theta, \phi)$-sector can be compactified by considering the quotient space $H^{2} / G . G$ is the discrete subgroup of the isometry group $\mathrm{SO}(2,1)$ of $H^{2}$. If one requires this space to be orientable, it becomes a Riemann surface of genus $g>1$. Genus one is a torus and a higher degree genus is a hyperelliptic surface $y^{2}=P(x)$, where $\mathrm{P}$ is a complex polynomial of degree $2 g+1$. The topology of the manifold is $\mathbb{R}^{2} \times S_{g}$.

There are several possibilities to find black holes with non-trivial topologies. With $c=0$ and $k=\Lambda / 3<0$ one has an uncharged static topological $\mathrm{BH}$ solution in AdS gravity. Secondly, one may set $k=0$ which gives a space not asymptotically AdS. For $c>0$ and $-1 \leq 3 b c<2$ it is a $\mathrm{BH}$. The condition $3 b c=-1$ gives an extreme $\mathrm{BH}$. The scalar curvature in case $k=0$ is

$$
R=-6 c / r(1+b / r)
$$

which is singular at $r=0$, but for $r \rightarrow \infty R$ vanishes. Thirdly, one may set $b=2 / 3 c-\eta$ and $k=\Lambda / 3>0$ in (8.5). In the limit $c \rightarrow 0$ on gets for $V$

$$
V(r)=1-2 \eta / r-\Lambda r^{2} / 3
$$

This spacetime is similar to the Schwarzschild-de Sitter solution but with nontrivial topology. As $r \rightarrow \infty$ the scalar curvature $S \rightarrow 4 \Lambda$. (8.8) is not a solution of Einstein's Equation (8.8) has two zeroes $r_{-}<r_{+} \cdot r_{-}$is the black hole event horizon radius and $r_{+}$is a cosmological horizon. Finally, one can obtain a toroidal black hole spacetime using another analytic continuation

$$
\tau=\sqrt{d}, \rho=r / \sqrt{d}, \Theta=\theta \sqrt{d}, \Phi=\phi, \beta=b / \sqrt{d}, \gamma=c / \sqrt{d}
$$

In the limit $d \rightarrow 0$ one has

$$
\mathrm{d} s^{2}=-V(r) \mathrm{d} t^{2}+V(r)^{-1} \mathrm{~d} r^{2}+r^{2}\left(\mathrm{~d} \theta^{2}+\theta^{2} \mathrm{~d} \phi^{2}\right)
$$

with

$$
V(r)=3 b^{2} c / r-3 b c+c r-k r^{2}
$$

Depending on the parameter values (8.10) may represent a black hole. The angular sector has a flat metric $\mathrm{d} \sigma^{2}=r^{2}\left(\mathrm{~d} \theta^{2}+\theta^{2} \mathrm{~d} \phi^{2}\right)$. Changing coordinates to Cartesian ones with

$$
x \simeq x+n, \quad y \simeq y+m, n, m \in Z
$$

on gets a compact orientable surface, a torus, with a topology $\mathbb{R}^{2} \times S^{1} \times S^{1}$. Putting $c=-2 \eta / L, b=\sqrt{L / 3}$, and $k=\Lambda / 3$ and letting $L \rightarrow \infty$ on has

$$
V(r)=-2 \eta / r-\Lambda r^{2} / 3
$$

This is for $\eta>0$ an uncharged static toroidal black hole, known in AdS gravity. For $k \geq 0$ the black hole interpretation is lost since (8.11) has no real root for $k=0$ and only one real root for positive $k$ which is not a black hole event horizon.

For different genus values one has: $g=1$, a torus, only solutions with asymptotically AdS. For $g>1$ dS BHs exist. Interestingly, Weyl conformal and AdS gravity alone have consistent interaction with massless higher spin fields. 
I propose a toy model for generic black hole structure, or constituents, in which the hole consist of tori of decreasing sizes starting from the radius of the hole. The next torus radius is the previous torus tube radius increasing the complexity of the hole topology. The scale dependence of this spacetime structure should be studied by this scale method.

\section{Conclusions}

The elegance and power of general relativity are realized when the basic Einstein equations are generalized to the largest local symmetry groups including the Weyl conformal symmetry and the full Poincaré symmetry with torsion of spacetime. The statement "to modify it [EG] without destroying the whole structure seems to be impossible" did not turn out to be true, if the modification is done properly. The main conclusion of this study is that local conformal symmetry allows us to obtain a unified description of gravity and the standard model. All interactions are described in geometrical or geometrized formalism which contains the familiar SM quantum particles. A possible model for matterspacetime unification was reviewed in Subsection 3.2. I proposed in Section 8 a toy model for black hole structure, or constituents, in which the holes consist of tori of decreasing sizes starting from the radius of the hole.

With conformal symmetry the applicability of GR is greatly expanded. The cosmological picture of the universe is changed substantially as became clear several decades later [2] [44] [45]. The "one start" big bang is replaced by the cyclic picture of the universe. Conformal symmetry makes it possible to geometrize all other interactions, of which electromagnetism received in Section 4 special attention. Further it has been shown that dark matter of galaxies can be explained with the different velocity profile of conformal equations [17]. A theory for dark energy has also been proposed [18]. At high energy/matter density, like inside black holes, the pure massless conformal symmetry must be modified to include mass of Dirac fields. Torus solutions in theories with massive Dirac field in the presence of torsion are interesting. Most likely, torsion is a neglected important sector of gravity. Interesting structures in spacetime with certain area and volume quantization methods should be restudied. The area and volume of a torus are $A=4 \pi^{2} r R$ and $V=2 \pi^{2} r^{2} R$ where $r$ is the "tube" radius and $R$ is the radius of the "tube" center line. To make the torus area compatible with ball area requires $r=$ const $\times R$.

\section{Acknowledgements}

I thank Dr. William Straub for correspondence and comments on the manuscript.

\section{References}

[1] Bars, I., Steinhardt, P. and Turok, N. (2013) Local Conformal Symmetry in Physics and Cosmology. Physical Review D, 89, Article ID: 043515. arXiv:1307.1848.

[2] Bars, I., Steinhardt, P. and Turok, N. (2013) Cyclic Cosmology, Conformal Symme- 
try and the Metastability of the Higgs. Physics Letters $B, 726,50-55$. arXiv: 1307.8106. https://doi.org/10.1016/j.physletb.2013.08.071

[3] Campiglia, M., Gambini, R. and Pullin, J. (2016) Conformal Loop Quantum Gravity Coupled to the Standard Model. arXiv:1609.04028.

[4] Harari, H. (1984) Composite Models for Quarks and Leptons. Physics Reports, 104, 159-179. https://doi.org/10.1016/0370-1573(84)90207-2

[5] Raitio, R. (1980) A Model of Lepton and Quark Structure. Physica Scripta, 22, 197198. https://doi.org/10.1088/0031-8949/22/3/002

[6] Raitio, R. (2016) Combinatorial Preon Model for Matter and Unification. Open Access Library Journal, 3, e3032. https://doi.org/10.4236/oalib.1103032

[7] Raitio, R. (2016) Standard Model Matter Emerging from Spacetime Preons. Open Access Library Journal, 3, e2788. https://doi.org/10.4236/oalib.1102788

[8] Raitio, R. (2016) A Conformal Preon Model. Open Access Library Journal, 3, e3262. https://doi.org/10.4236/oalib.1103262

[9] Weyl, H. (1918) Sitzsungber. Preussischen Akademie der Wissenschaften, 465-480.

[10] Einstein, A. and Rosen, N. (1935) The Particle Problem in the General Theory of Relativity. Physical Review, 48, 73-77. https://doi.org/10.1103/PhysRev.48.73

[11] Cartan, É. (1923) (1924) et (1925) Sur les variétés á connexion affine et la théorie de la relativité généralisée. Annales scientifiques de PÉcole Normale Supérieure, 42, 17-88.

[12] Rovelli, C. (2011) Zakopane Lectures on Loop Gravity. arXiv:1102.3660.

[13] Carroll, S. (2004) Spacetime and Geometry: An Introduction to General Relativity. Addison-Wesley, San Francisco.

[14] Mannheim, P. (2007) Solution to the Ghost Problem in Fourth Order Derivative Theories. Foundations of Physics, 37, 532-571. arXiv:hep-th/0608154.

https://doi.org/10.1007/s10701-007-9119-7

[15] Bender, C. and Mannheim, P. (2008) Giving up the Ghost. Journal of Physics A, 41, Article ID: 304018. arXiv:0807.2607. https://doi.org/10.1088/1751-8113/41/30/304018

[16] Mannheim, P. (2012) Making the Case for Conformal Gravity. Foundations of Physics, 42, 388-420. arXiv:1101.2186. https://doi.org/10.1007/s10701-011-9608-6

[17] Mannheim, P. (2006) Alternatives to Dark Matter and Dark Energy. Progress in Particle and Nuclear Physics, 56, 340-445. astro-ph/0505266. https://doi.org/10.1016/j.ppnp.2005.08.001

[18] Mannheim, P. (2016) Mass Generation, the Cosmological Constant Problem, Conformal Symmetry, and the Higgs Boson. arXiv:1610.08907.

[19] Greenberg, O. (1964) The Color Charge Degree of Freedom in Particle Physics. arXiv:0805.0289.

[20] Bekenstein, J. (1972) Non Existence of Baryon Number for Static Black Holes I and II. Physical Review D, 5, 1239-1246. https://doi.org/10.1103/PhysRevD.5.1239

[21] Wheeler, J. (1971) Cortona Symposium on Weak Interactions. Accademia Nazionale dei Lincei, Rome.

[22] Fabbri, L. (2008) Higher Order Theories of Gravitation, PhD Thesis. arXiv: 0806.2610.

[23] Lanczos, C. (1938) A Remarkable Property of the Riemann-Christoffel Tensor in four Dimensions. Annals of Mathematics, 39, 842-850.

https://doi.org/10.2307/1968467 
[24] Maldacena, J. (2011) Einstein Gravity from Conformal Gravity. arXiv:1105.5632.

[25] Anastasiou, G. and Olea, R. (2016) From Conformal to Einstein Gravity. Physical Review D, 94, Article ID: 086008. arXiv:1608.07826. https://doi.org/10.1103/physrevd.94.086008

[26] Coleman, S. (1988) Aspects of Symmetry: Selected Erice Lectures. Cambridge University Press, Cambridge.

[27] Ariwahjoedi, S., Astuti, V., Kosasih, J., Rovelli, C. and Zen, F. (2016) Statistical Discrete Geometry. arXiv:1607.08629.

[28] Barbero G., J.F. and Perez, A. (2015) Quantum Geometry and Black Holes. arXiv:1501.02963.

[29] Mannheim, P. (2014) PT Symmetry, Conformal Symmetry, and the Metrication of Electromagnetism. arXiv:1407.1820.

[30] Mannheim, P. (2016) Conformal Invariance and the Metrication of the Fundamental Forces. arXiv:1603.08405.

[31] Fabbri, L. (2014) Conformal Standard Model. General Relativity and Gravitation, 44, 3127-3138. arXiv:1107.0466. https://doi.org/10.1007/s10714-012-1440-6

[32] Brans, C. and Dicke, R. (1961) Mach's Principle and a Relativistic Theory of Gravitation. Physical Review, 124, 925-935. https://doi.org/10.1103/PhysRev.124.925

[33] Fabbri, L. (2014) Metric-Torsional Conformal Gravity. Physics Letters B, 707, 415-417. arXiv:1101.1761. https://doi.org/10.1016/j.physletb.2012.01.008

[34] Fabbri, L. (2013) Conformal Gravity with Dirac Matter. Annales de la Fondation Louis de Broglie, 38, 155-165. arXiv:1101.2334.

[35] Shapiro, I (2002) Physical Aspects of the Space-Time Torsion. Physics Reports, 357, 113-213. hep-th/0103093. https://doi.org/10.1016/s0370-1573(01)00030-8

[36] Trautman, A. (2006) The Einstein-Cartan Theory. In: Francoise, J.-P., Naber, G.L. and Tsou, S.T., Eds., Encyclopedia of Mathematical Physics, Elsevier, Amsterdam, Vol. 2, 189-195. https://doi.org/10.1016/B0-12-512666-2/00014-6

[37] Kibble, T. (1961) Lorentz Invariance and the Gravitational Field. Journal of Mathematical Physics, 2, 212-221. https://doi.org/10.1063/1.1703702

[38] Sciama, D. (1962) On the Analogy between Charge and Spin in General Relativity. In: Casciaro, B., Ed., Recent Developments in General Relativity, Pergamon Press, Oxford, 415-439.

[39] Sciama, D. (1964) The Physical Structure of General Relativit. Reviews of Modern Physics, 36, 463-469. https://doi.org/10.1103/RevModPhys.36.463

[40] Poplawski, N. (2011) Cosmological Constant from Quarks and Torsion. Annalen der Physik, 523, 291-295. https://doi.org/10.1002/andp.201000162

[41] Degrassi, G., Di Vita, S., Elias-Miro, J., Espinosa, J., Giudice, G., Isidori, G. and Strumia, A. (2012) Higgs Mass and Vacuum Stability in the Standard Model at NNLO. JHEP, 1208, 098. arXiv:1205.6497.

[42] Bars, I., Chen, S.-H., Steinhardt, P. and Turok, N. (2012) Complete Set of Homogeneous Isotropic Analytic Solutions in Scalar-Tensor Cosmology with Radiation and Curvature. Physical Review D, 86, Article ID: 083542. arXiv:1207.1940. https://doi.org/10.1103/PhysRevD.86.083542

[43] Klemm, D. (1998) Topological Black Holes in Weyl Conformal Gravity. Classical and Quantum Gravity, 15, 3195-3201. gr-qc/9808051. https://doi.org/10.1088/0264-9381/15/10/020

[44] Penrose, R. (2016) Fashion, Faith, and Fantasy in the New Physics of the Universe. 
Princeton University Press, Princeton. https://doi.org/10.1515/9781400880287

[45] Gielen, S. and Turok, N. (2016) Quantum Propagation across Cosmological Singularities. arXiv:1612.02792.

Submit or recommend next manuscript to OALib Journal and we will provide best service for you:

- Publication frequency: Monthly

- 9 subject areas of science, technology and medicine

- Fair and rigorous peer-review system

- Fast publication process

- Article promotion in various social networking sites (LinkedIn, Facebook, Twitter, etc.)

- Maximum dissemination of your research work

Submit Your Paper Online: Click Here to Submit

Or Contact service@oalib.com 